\title{
Quantitative Understanding of Nanoparticle Uptake in Watermelon Plants
}

\author{
Ramesh Raliya ${ }^{1}$, Christina Franke ${ }^{1,2}$, Sanmathi Chavalmane ${ }^{1}$, Remya Nair ${ }^{1}$, Nathan Reed \\ and Pratim Biswas ${ }^{1 *}$ \\ ${ }^{1}$ Department of Energy, Environmental and Chemical Engineering, Washington University in St. Louis, St. Louis, MO, USA, \\ ${ }^{2}$ Department of Biomedical Engineering, Case Western Reserve University, Cleveland, OH, USA
}

\section{OPEN ACCESS}

Edited by:

David W. M. Leung, University of Canterbury, New Zealand

Reviewed by:

Dharmendra Kumar Gupta, Estación Experimental del Zaidin-The Spanish National Research Council (CSIC), Spain John L. Freeman, Intrinsyx Technologies

Corporation, USA

*Correspondence:

Pratim Biswas

pbiswas@wustl.edu

Specialty section:

This article was submitted to

Plant Traffic and Transport, a section of the journa

Frontiers in Plant Science

Received: 25 March 2016 Accepted: 12 August 2016 Published: 26 August 2016

Citation:

Raliya R, Franke C, Chavalmane S, Nair R, Reed N and Biswas P (2016) Quantitative Understanding of Nanoparticle Uptake in Watermelon Plants. Front. Plant Sci. 7:1288 doi: 10.3389/fpls.2016.01288
The use of agrochemical-nutrient fertilizers has come under scrutiny in recent years due to concerns that they damage the ecosystem and endanger public health. Nanotechnology offers many possible interventions to mitigate these risks by use of nanofertilizers, nanopesticides, and nanosensors; and concurrently increases profitability, yields, and sustainability within the agricultural industry. Aerosol based foliar delivery of nanoparticles may help to enhance nanoparticle uptake and reduce environmental impacts of chemical fertilizers conventionally applied through a soil route. The purpose of this work was to study uptake, translocation, and accumulation of various gold nanostructures, $30-80 \mathrm{~nm}$, delivered by aerosol application to a watermelon plant. Cellular uptake and accumulation of gold nanoparticles were quantified by Inductively Coupled Plasma-Mass Spectroscopy (ICP-MS). Observations suggested that nanoparticles could be taken up by the plant through direct penetration and transport through the stomatal opening. Observed translocation of nanoparticles from leaf to root shows evidence that nanoparticles travel by the phloem transport mechanism. Accumulation and transport of nanoparticles depend on nanoparticle shape, application method, and nature of plant tissues.

Keywords: aerosol delivery, biointerface, gold nanoparticle, internalization, nanoparticle, watermelon

\section{INTRODUCTION}

The application of nanotechnology in the area of plant sciences has been extensively studied in recent years (Peng et al., 2012; Chen et al., 2014). A key aspect of the work of plant nutritional scientists is toward the precise delivery of nutrients and enhanced nutrient uptake. Chemical fertilizer uptake efficiency in plants is low due to fixation of nutrients with other soil composites or run off due to precipitation leading to a growing anthropogenic eutrophication issue (Smith et al., 1999; Hautier et al., 2014). To address these challenges, nanoparticles have been researched for use as insecticides (Wibowo et al., 2014), fungicides (Becker et al., 2010; Capaldi Arruda et al., 2015; Saharan et al., 2015) and nanofertilizers (DeRosa et al., 2010; Raliya and Tarafdar, 2013; Liu and Lal, 2014, 2015; Raliya et al., 2014, 2016; Tarafdar et al., 2014). While there has been a significant push to engineer beneficial nanoparticles, there are also unintentional exposures and releases of nanoparticles from various industries that may end up in the ecosystem (Biswas and Wu, 2005; Yang et al., 2014). It is well reported that internalization and subsequent toxicity of nanoparticles within in vitro microenvironments depend on chemical composition, particle size, and surface chemistry that affects ligand-receptor interactions. For instance, Jiang et al. (2008) established size 
dependent reactive oxygen species (ROS) generation caused by $\mathrm{TiO}_{2}$ nanoparticles. The highest ROS activity per unit area was observed for $30 \mathrm{~nm}$ particles, and observed to be constant above $30 \mathrm{~nm}$. Similarly, Albanese et al. (2012) provide a rationale by correlating the properties of nanomaterials such as size, shape, chemical functionality, surface charge, and composition with biomolecular signaling, biological kinetics, transport and toxicity in cell culture studies. However, there is no study that correlates morphology dependent nanoparticle uptake to an application method.

Nair et al. (2010) and González-Melendi et al. (2008) reviewed the delivery of nanoparticulate materials to plants and provided evidence of internalization by various microscopic techniques. Unfortunately, most microscopic imaging only allows for a qualitative investigation of a minute fraction of plant tissues ranging between nanometer to micrometer size scale. Further biasing can occur during staining of plant intracellular bodies for electron microscopy due to nanomaterial losses. Therefore, the tiny fraction of plant tissue examined may not necessarily represent the whole plant. Consequently, interactions of nanoparticles with plants are possible in many ways, and their impact on crop plant and food safety need to be investigated systemically.

Inductively coupled plasma-mass spectroscopy (ICP-MS) or inductively coupled plasma-optical emission spectroscopy (ICP-OES) are powerful tools to analyze metal accumulation in plants. In both the techniques, the entire plant tissue digested in the acid mixture before analysis. ICP-MS is preferred for nanoparticle detection in biological samples, due its detection sensitivity (Scheffer et al., 2008). During ICP-MS analysis, digested samples are introduced into argon plasma as aerosol droplets. The droplets dry in the plasma region, dissociate into the molecular constituents, and then form singly-charged ions, which are directed to a filtering device known as the mass spectrometer.

In the present study, gold nanoparticles (Au NPs) were used due to their biologically inert properties and their past utilization in genetic engineering through adsorbtion of DNA on a gold particle surface which can be delivered to cells (Ghosh et al., 2008; Thakor et al., 2011). Au NPs have shown great promise in the facile, on-site detection of contagious plant viruses when applied to a strip sensor (Zhao et al., 2011; Wei et al., 2014). These nanoparticles can be functionalized to target specific sites within an organism's cellular substructure, and could allow genetic modifications to be made from within the plant itself, rather than through a traditional in-vitro process. Moreover, if dispersed throughout a plant, Au NPs may eventually prove useful for real-time plant disease detection. Nonetheless, it is essential to understand the fundamental mechanisms of morphologydependent cellular uptake of gold nanoparticles, as well as their transport and subsequent fate in a plant system.

In this work, we describe the effects of gold nanostructure on their internalization, translocation and accumulation in the watermelon plant. Au NPs of various sizes and morphologies were synthesized, characterized, and applied to the plants in aqueous suspension via foliar (drop-cast and aerosol) application. The objectives of this study were (1) to optimize nanoparticle delivery in plants comparing conventional drop cast approach v/s aerosol technique (2) to understand the uptake, transport and accumulation of nanoparticles in correlation to nanoparticle morphology and (3) to develop an alternative approach of nanoparticle quantification in plant tissue using ICP-MS. Au NPs were chosen as a representative of metallic nanoparticles because of its broad application and ease to tune particle morphology. Watermelon plant (Citrullus lanatus) was chosen because of its popularity as an edible fruit around the world, and also because it has leaves with both large stomata and vessel size, which may facilitate nanoparticle uptake and translocation.

\section{MATERIALS AND METHODS}

The experimental plan of the study is summarized in Table $\mathbf{1}$, and details of the same are described in the following sections.

\section{Gold Nanoparticle Synthesis and Characterization \\ Reagents}

Ascorbic acid (99.8\%) was procured from J.T. Baker. Hexadecyltrimethyl ammonium chloride (>95.0\%) was purchased from Tokyo Chemical Industry Co, Ltd. odium borohydride ( $\geq 99 \%)$, hexadecyltrimethyl ammonium bromide

TABLE 1 | Test plan for the overall experiment.

\begin{tabular}{|c|c|c|c|c|}
\hline No. & Experiment & Objective & Investigation Finding & Notes \\
\hline 1 & Synthesis of Au nanostructures & $\begin{array}{l}\text { To produce nanostructures with varying } \\
\text { morphologies }\end{array}$ & $\begin{array}{l}\text { Sphere, cube, rhombic decahedral, } \\
\text { and rod shape Au nanostructures were } \\
\text { synthesized in the range of 30-90 nm }\end{array}$ & $\begin{array}{l}\text { Morphology of Au nanostructures we } \\
\text { confirmed by UV-VIS spectroscopy, }\end{array}$ \\
\hline 2 & $\begin{array}{l}\text { Application of synthesized } \\
\text { nanostructures to plants }\end{array}$ & $\begin{array}{l}\text { To study Au nanostructure-watermelon } \\
\text { plant interaction }\end{array}$ & $\begin{array}{l}\text { Aqueous suspensions of AuNPs were } \\
\text { diluted to } 100 \text { ppm and applied to } \\
\text { watermelon leaves }\end{array}$ & $\begin{array}{l}\text { Two foliar application methods were } \\
\text { aerosol and drop-cast methods }\end{array}$ \\
\hline 3 & $\begin{array}{l}\text { Elemental analysis of Au } \\
\text { concentrations in plant }\end{array}$ & $\begin{array}{l}\text { To compare the efficacy of uptake, } \\
\text { translocation and accumulation of } \\
\text { various Au nanostructures }\end{array}$ & $\begin{array}{l}\text { Spherical particles translocated to } \\
\text { greatest extent by aerosol method; Rods } \\
\text { translocated to greatest extent by } \\
\text { drop-cast method }\end{array}$ & $\begin{array}{l}\text { Elemental analysis performed using } \\
\text { ICP-MS }\end{array}$ \\
\hline
\end{tabular}


( $\geq 99.0 \%$ ), sodium bromide ( $\geq 99.0 \%$ ), silver nitrate (99.9999\%), gold (III) chloride trihydrate $(\geq 99.9 \%)$, and gold chloride solution $(200 \mathrm{mg} / \mathrm{dL})$ were purchased from Sigma-Aldrich. All chemicals were used as received.

\section{Synthesis}

Gold nanoparticles of spherical, cubic, rhombic dodecahedral (RD), and rod morphologies were prepared using seed-mediated methods (Becker et al., 2010; Wu et al., 2010). Gold seeds (2$3 \mathrm{~nm}$ ) were produced through the reaction of gold chloride with the reductant, ascorbic acid. Formation of gold seed particles was evident due to the immediate brown color formation upon addition of catalyst, sodium borohydride. Au NPs formation occurs as a result of the nucleation of gold seeds in growth solutions, varying the amount of gold seeds and concentration of reducing agent.

\section{Characterization}

Synthesized nanoparticles were characterized for their morphological properties prior to their application to the watermelon plants.

\section{Transmission Electron Microscopy (TEM)}

Nanoparticle samples were prepared for TEM characterization by placing a drop of aqueous particle suspension on a carbon-coated copper grid and allowing the sample to air-dry. TEM images were viewed and obtained with the aid of a Tecnai $G^{2}$ Spirit transmission electron microscope (FEI, USA).

\section{UV-visible (UV-Vis) Spectrophotometry}

Nanoparticle samples were diluted 2X with deionized (DI) water for UV-Visible spectrophotometry analyses. Surface plasmon resonance of each sample was determined using a Varian Cary 50 UV-visible spectrophotometer (Varian, Inc., USA), measuring the absorbance of light with wavelengths in the $400-1000 \mathrm{~nm}$ range at medium sample speed. The maximum peak in each absorbance band was taken to be the surface plasmon resonance of the sample.

\section{Dynamic Light Scattering (DLS)}

Samples were prepared for DLS characterization by diluting $2 \mathrm{X}$ with DI water. Using DLS, hydrodynamic size and zeta potential measurements were performed using a Malvern Zeta Sizer Nano ZS (Malvern Instruments, USA). Samples were equilibrated for $2 \mathrm{~min}$ at $25^{\circ} \mathrm{C}$ before obtaining each measurement. All the measurements were performed in triplicate.

\section{Inductively-Coupled Mass Spectrometry (ICP-MS)}

To determine the nanoparticle concentration in suspension, 100 $\mu \mathrm{L}$ of each nanoparticle solution was digested in $400 \mu \mathrm{L}$ aqua regia (Nitric acid: hydrochloric acid, 3:1). Digested samples were diluted with $4.5 \mathrm{~mL} 1 \%$ nitric acid. Elemental concentration of gold in each sample was measured with the aid of an ELAN DRC II ICP-MS (Perkin Elmer, Inc., USA). The concentrations of spheres, cubes, $\mathrm{RD}$, and rods (in ppm) were $2.29 \times 10^{3}, 1.12 \times$ $10^{4}, 1.18 \times 10^{4}$, and $1.44 \times 10^{3}$, respectively (Table 2).

\section{Watermelon Plants and Growth Conditions}

All plants were grown in a controlled environment chamber with a constant temperature of $34 \pm 2{ }^{\circ} \mathrm{C}, 60 \pm 2 \%$ relative humidity, constant air flow, a photoperiod of $16: 8 \mathrm{~h}$ and a photosynthetic photon flux density of $750 \mu \mathrm{mol} \mathrm{m}^{-2} \mathrm{~s}^{-1}$. Black Diamond watermelon (C. lanatus) seeds were grown in plastic pots (38 mm square) filled with moisture control potting mix (Miracle-Gro Lawn Products, USA). One seed was planted in each pot. Soil was kept moist and pots were covered until seed germination. Upon germination, pot covers were removed and seedlings were given $10 \mathrm{ml}$ deionized water daily. In addition, 5 $\mathrm{mL}$ of nutrient solution mixture (Miracle Grow, USA) was also supplied on every alternate day.

\section{Exposure of Nanoparticles to Plant}

Each nanoparticle sample was diluted with DI water to $100 \mathrm{ppm}$ for spraying. To prepare watermelon plants for spraying, all leaves were left intact, but one was removed to function as a control. Experiments were performed in triplicate for each type of particle and each application method, and four plants, each of 14 days old were used for control, on which only DI water was

TABLE 2 | Characterization data for nanoparticles in aqueous suspension.

\begin{tabular}{|c|c|c|c|c|c|c|}
\hline Particle Morphology & Particle Size $^{\mathrm{a}}(\mathrm{nm})$ & Hydrodynamic Size ${ }^{c}(\mathrm{~nm})$ & $P D I^{c, d}$ & $\lambda_{\max }{ }^{\mathrm{e}}(\mathrm{nm})$ & Zeta Potential $^{\mathrm{C}}(\mathrm{mV})$ & $\begin{array}{c}\text { Concentration, } \\
\mathrm{Au}(\mathrm{ppm})\end{array}$ \\
\hline Sphere & $35 \pm 4.2^{*}$ & $61.0 \pm 1.6$ & $0.457 \pm 0.02$ & 527 & $-40.5 \pm 2.3$ & $2.29 \times 10^{3}$ \\
\hline Rhombic Dodecahedra & $65 \pm 3.4$ & $99.8 \pm 30.4$ & $0.066 \pm 0.01$ & 550 & $64.3 \pm 4.2$ & $1.18 \times 10^{4}$ \\
\hline Rod & $\begin{array}{l}20 \pm 0.92 \\
60 \pm 1.3^{b}\end{array}$ & $114.1 \pm 52.1$ & $0.296 \pm 0.03$ & 816 & $43.9 \pm 3.3$ & $1.44 \times 10^{3}$ \\
\hline Replicate $(n)$ & $n=50$ & $n=5$ & $n=5$ & - & $n=5$ & - \\
\hline
\end{tabular}

a Mean geometric diameter, obtained from TEM images.

${ }^{b}$ Diameter, length.

${ }^{c}$ From DLS, number based particle size distribution.

a Polydispersity index.

e Surface Plasmon Resonance, obtained from maximum UV-vis peak.

*Standard error from the mean value. 
applied. All the exposure experiments were conducted in between 11 a.m. and 1 p.m. to ensure stomatal opening.

\section{Aerosol Method}

A schematic of the atomizer application method is provided in Figure 1. The soil of the plants was covered so that no particles could be sprayed directly onto the soil. The surface of each plant's remaining mature leaf was sprayed for $10 \mathrm{~min}$ in a fume hood. Gold nanoparticles were aerosolized by a TSI atomizer under 30 psi flowing air.

During exposure, online measurements of the applied particle size and number concentration were monitored by Scanning Mobility Particle Sizer (SMPS, TSI, Inc., USA). Aerosol distributions are predominantly lognormal in character, so data is typically plotted on a lognormal $\mathrm{X}$-axis. In the simplest technique, particle data is plotted as a function of the concentration $(\mathrm{dN})$ for each particle size bin. The mode concentration of the size distribution is estimated by the concentration in the peak bin. $\mathrm{dN}$ (or $\Delta \mathrm{N}$ ) is the number of particles in the range (total concentration) and $\operatorname{dlog} \mathrm{Dp}$ (or $\Delta \log \mathrm{Dp}$ ) is the difference in the $\log$ of the channel width. dlogDp is calculated by subtracting the $\log$ of the lower bin boundary from the log of the upper boundary for each channel (normalizing for bin width). The concentration is divided by the bin width, giving a normalized concentration value that is independent of the bin width (TSI Inc., USA) using following formula by the TSI software.

$$
\mathrm{dN} / d \log D p=\frac{d N}{d \log D p}=\frac{d N}{\log D p, u-\log D p, l}
$$

Where,

$$
\begin{aligned}
d N & =\text { particle concentration } \\
D p & =\text { midpoint particle diameter } \\
D p, u & =\text { upper channel diameter } \\
D p, l & =\text { Lower channel diameter }
\end{aligned}
$$

\section{Drop-Cast Method}

In order to compare the nanoparticle uptake with respect to aerosol application, equal amount of each sample was placed in small droplets using auto pipette on the surface of a plant's mature leaf. Plants were left undisturbed after applying particles, allowing the droplets to air dry. An image of a leaf after treatment by the drop-cast method is provided in Figure 1.

\section{Nanoparticle Uptake, Transport, and Accumulation Analysis Using ICP-MS}

After nanoparticle exposure to plants either by an aerosol or drop cast method, plants were allowed to grow for $48 \mathrm{~h}$ in the environmental condition described above. This allowed accumulation of the nanoparticles as they were transported and interacted with plant cells and tissues.

\section{Harvest Plants}

Plants were harvested $48 \mathrm{~h}$ after applying the nanoparticles. To prepare plant samples for uptake analysis, roots were first washed with tap water to remove the adhered soil. The entire plant was then rinsed with DI water three times. Roots, stems, seed leaves, and sprayed leaves of each plant were separated into $20 \mathrm{ml}$ glass vials. The harvested samples were placed in a drying oven at $60^{\circ} \mathrm{C}$ until the dry matter reached a constant weight.

\section{Elemental Analysis}

After all samples had dried for $60-72 \mathrm{~h}$ at $60^{\circ} \mathrm{C}$, each sample was crushed into a fine powder. Powdered samples $(100 \mathrm{mg})$ were digested in $6 \mathrm{~mL}$ aqua regia $\left(\mathrm{HNO}_{3}\right.$ and $\left.\mathrm{HCl}\right)$ at $150^{\circ} \mathrm{C}$ using microwave digestion (CEM MARS 6 Xpress, CEM Corp., USA). After complete digestion, each sample was suspended in $5 \mathrm{~mL}$ DI water and filtered through a $25 \mathrm{~mm}$ syringe filter with a $0.45 \mu \mathrm{m}$ nylon membrane (VWR Inc., USA). These filtered samples were analyzed with the aid of an ELAN DRC II ICP-MS (Perkin Elmer, Inc., USA) to determine the concentration of elemental gold in each plant section. Based on the raw data of elemental detection

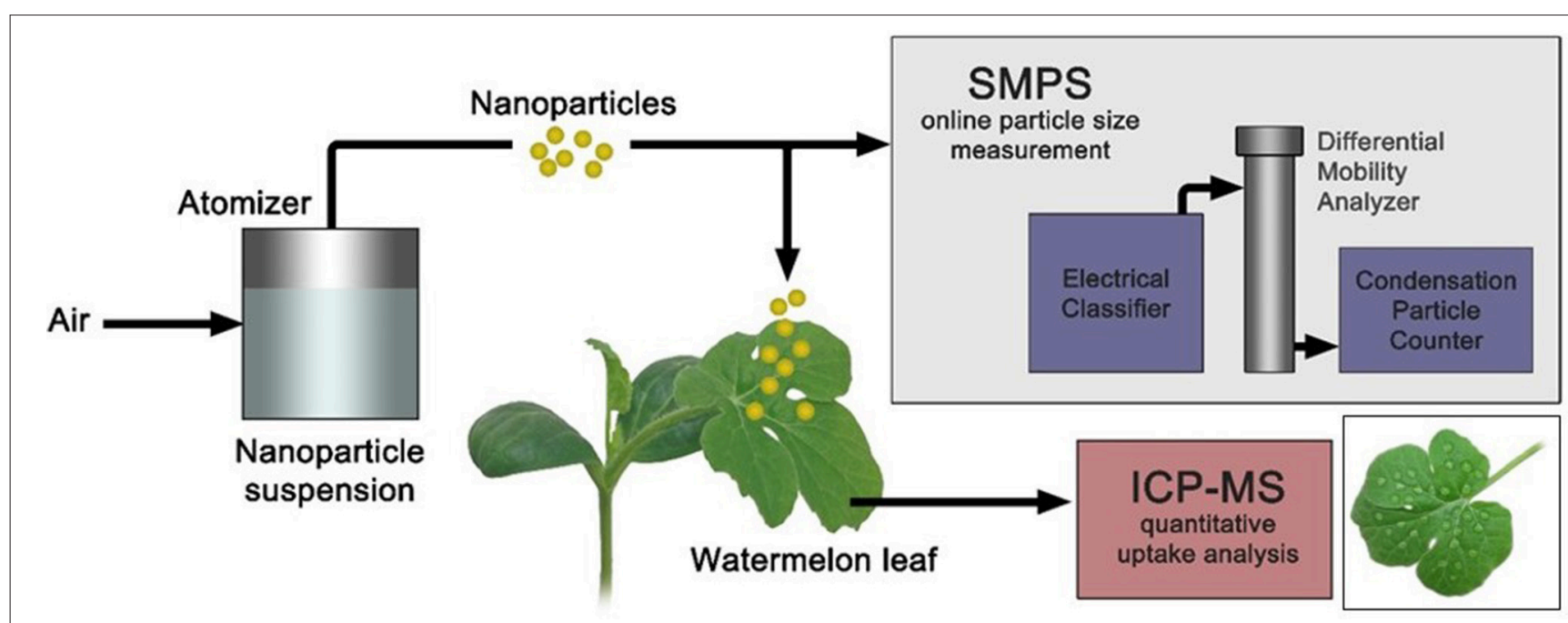

FIGURE 1 | Schematic of an aerosol method for nanoparticle delivery and quantification of nanoparticle uptake, accumulation, and translocation in watermelon. Bottom right image represents drop caste method of nanoparticle delivery to leaf using auto pipette. 
intensity, nanoparticles uptake and accumulation were calculated by the ELAN DRC II ICP MS software.

\section{Statistical Analyses}

The results were expressed as mean \pm SD (standard deviation); $n=5$ (except nanoparticle size data given in Table 2, where $n$ was 50). Statistical analyses was performed using data analyses function of Microsoft Excel V.2013. The significant difference in the same concentration of gold nanoparticle exposure (for individual shape) applied by drop-cast or aerosol method were analyzed by Student $t$-test. A $p$-value of less than $0.05(p<0.05)$ was considered as statistically significant.

\section{RESULTS AND DISCUSSION}

The particle characterization results are presented first, followed by a description of their impact on the plants. As indicated in test plan 1 (Table 1), particle morphologies, surface chemistry, and geometric diameters were determined to study their uptake and transport by watermelon (Figures 2A-D). Mean geometric diameters of spherical, cubic, and $\mathrm{RD}$ particles were found to be 35, 70, and $65 \mathrm{~nm}$ respectively (Table 2 ). Rod-shaped particles were $60 \mathrm{~nm}$ in length, $20 \mathrm{~nm}$ in diameter, and therefore with an aspect ratio of three (Table 2). The morphology and diameter of Au NPs were also evidenced by UV-Vis absorption spectra and specific color intensities of the solutions (Figures 2E,F).

Hydrodynamic size and zeta potential values were measured by the offline tool, DLS, and are summarized in Table 2. Zeta potential of the nanoparticles were determined to be $-40.5,56.7$, 64.3, and $43.9 \mathrm{mV}$ for spheres, cubes, RD, and rods respectively. The hydrodynamic sizes of all particles were larger than the geometric mean diameters determined from TEM images. This size disparity is due to the soft agglomeration of nanoparticles due to Van der Waals forces (Wang et al., 2013). Therefore, by comparing these two foliar application methods, we optimized the effect of applied droplet size for maximum Au NPs uptake in plants. In order to precisely determine nanoparticle delivery, the size distribution of the gold nanoparticles were monitored in real time using a (SMPS). Before each exposure, a background measurement of particle size distributions of air and DI water was carried out. The results (base lines in the Figure 3B) show that DI water and air do not have any significant particle concentration or formation, thus implying a negligible effect on the aerosol size and number concentration measured by the SMPS. The SMPS measurements showed good agreement with the geometric mean diameter (GMD) observed by TEM (Figures 3A,B). The observations indicate that shear forces in the atomizer during

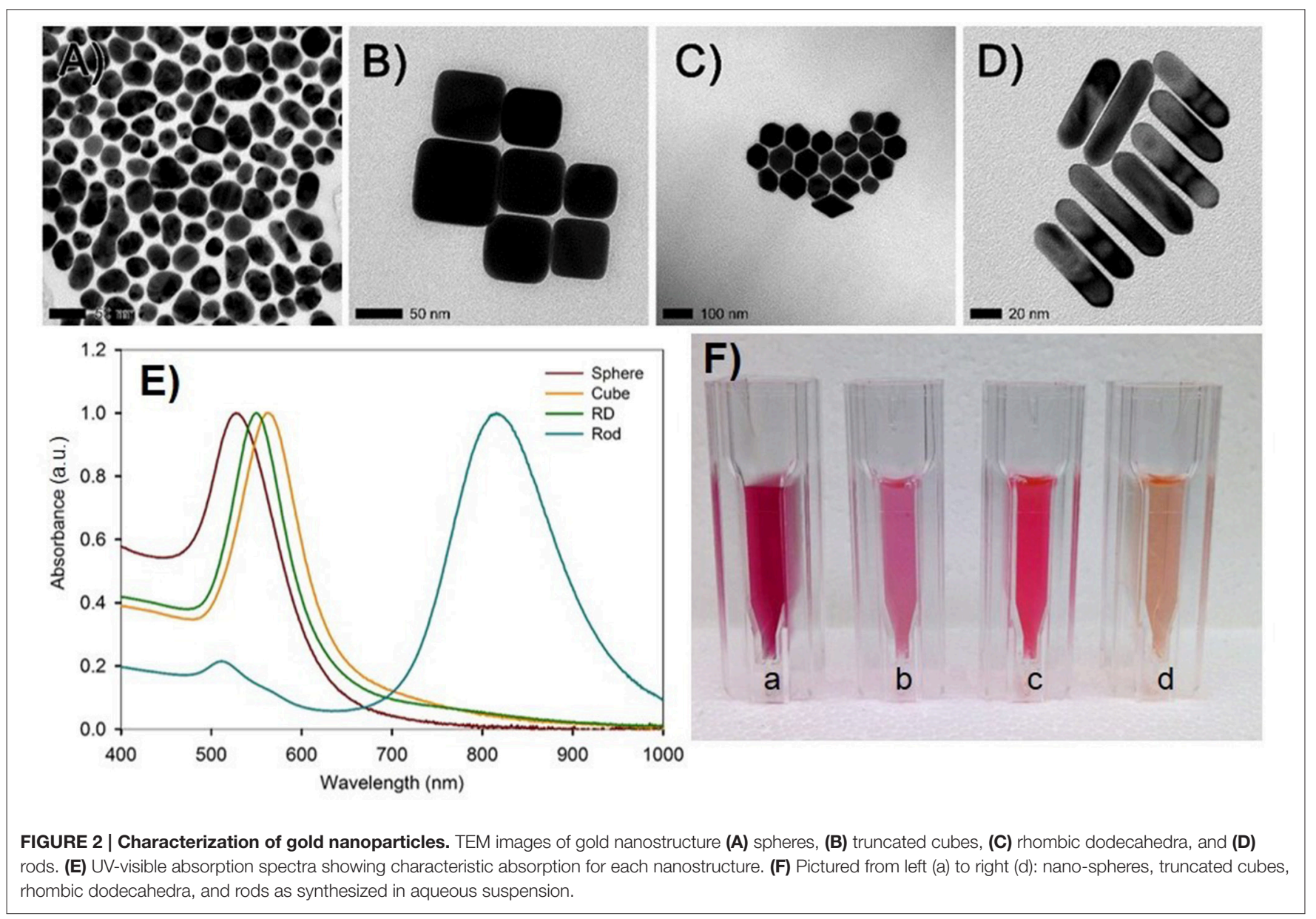


aerosolization resulted in the break-up of Au NP agglomerates and the formation of singlet particles (Brandt et al., 1987).

The nanoparticle suspensions were aerosolized and delivered to the watermelon leaf surface. For comparison, a solution drop method was employed concurrently for all the sample material in equal exposure concentration and volume. After $48 \mathrm{~h}$ all the samples were harvested and analyzed by ICP-MS to detect $\mathrm{Au}$ NPs concentration in leaves, stem and roots. The measured concentrations of elemental gold in each sample were normalized by the dried mass of the plant section, and this data was processed to determine the percent of recovered gold in each plant section.

ICP-MS analyses suggest that, when applied by drop-cast method, $28.0 \%$ of spherical particles were accumulated in the leaf, followed by $58.6 \%$ recovered in the stem and $13.4 \%$ in the root (Table 3). Comparing the results of the two application methods, aerosol application of low aspect ratio particles (sphere, cube, and rhombic dodecahedra) enhanced their transport, resulting in a greater percentage of gold being recovered in the root sections. For sphere, cube, and RD morphologies, the increase in translocation rate of the aerosolized vs. drop-cast nanoparticles was $10.9,2.3$, and $11.3 \%$ respectively, whereas a $37.4 \%$ decrease in translocation rate was observed for the nanorods (Table 3 ). This trend suggests that the smaller droplet size delivered by the aerosol method increased translocation of low aspect ratio particles to the plant roots, but inhibited the translocation of higher aspect ratio nanoparticles. The opposite trend was observed for the larger droplet size delivered by the dropcast method. When the nanoparticles were applied using the drop cast method, the rods, which had a higher aspect ratio than other morphologies tested, translocated to the roots to the greatest extent $(49.2 \%)$ as compared to the translocation of spheres (13.4\%), cubes (7.3\%), and rhombic dodecahedra (8.3\%; Figures $4 \mathrm{~A}-\mathrm{C})$. Thus, the larger droplet size may improve the uptake and transport of high aspect ratio nanoparticles.

Furthermore, trends comparing all nanoparticles were identified. For drop-cast application methods, a trend in the efficacy of translocation (percent of recovered gold accumulated in roots) was observed as: $\operatorname{rod}(49.3 \%)>$ sphere $(13.4 \%)>\mathrm{RD}$
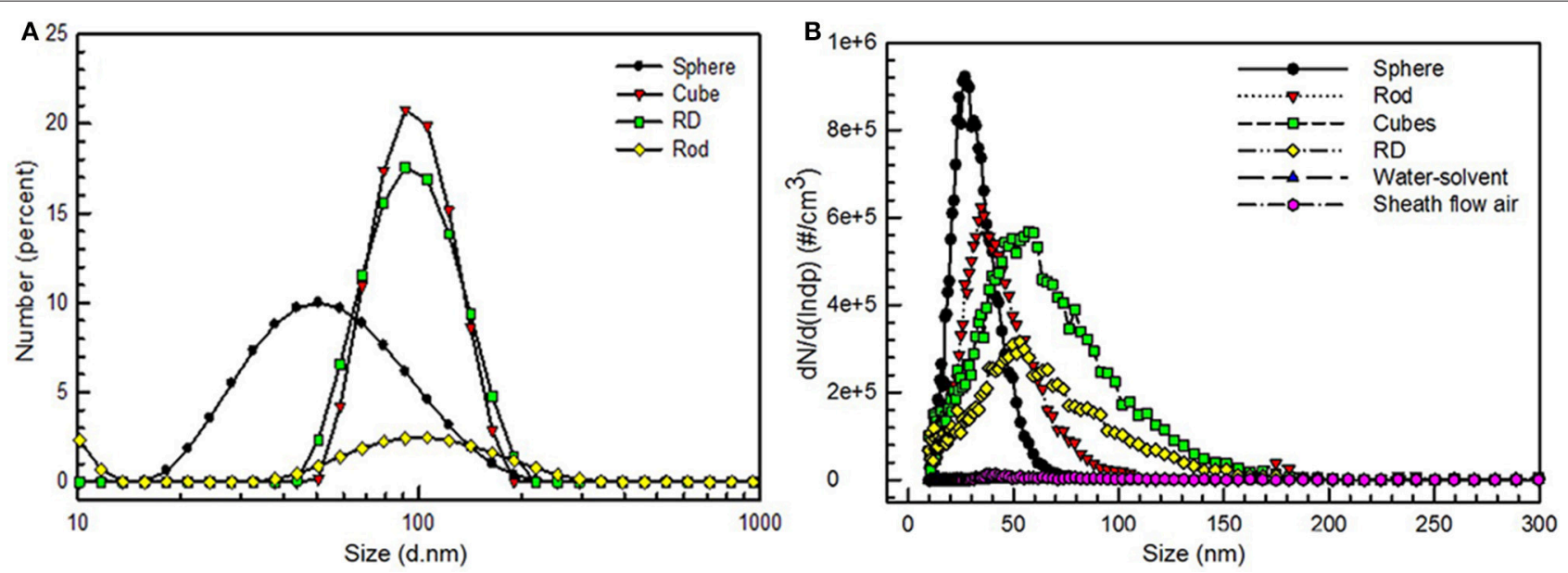

FIGURE 3 | Online and offline size measurement of gold nanostructures. (A) Hydrodynamic size measurements performed using light scattering (DLS) technique. (B) Online measurements of number concentrations of particles, monitored by Scanning Mobility Particle Sizer (SMPS).

TABLE 3 | Elemental analysis results of gold in plant sections by ICP-MS.

\begin{tabular}{|c|c|c|c|c|c|}
\hline Particle Morphology & Method & Amount in leaf $(\%)$ & Amount in stem (\%) & Amount in root $(\%)$ & $\begin{array}{c}\text { Mean percent difference } \\
\text { (drop-cast vs aerosol) in root }\end{array}$ \\
\hline \multirow[t]{2}{*}{ Sphere } & Drop-cast & $28.0 \pm 1.3^{a}$ & $58.6 \pm 2.7$ & $13.4 \pm 0.3$ & 10.9 \\
\hline & Aerosol & $65.5 \pm 1.7$ & $10.1 \pm 0.1$ & $24.4 \pm 1.1$ & \\
\hline \multirow[t]{2}{*}{ Cube } & Drop-cast & $57.9 \pm 2.6$ & $34.8 \pm 1.7$ & $7.3 \pm 0.5$ & 2.3 \\
\hline & Aerosol & $60.6 \pm 0.8$ & $29.8 \pm 0.9$ & $9.6 \pm 0.1$ & \\
\hline \multirow[t]{2}{*}{ Rhombic dodecahedra } & Drop-cast & $52.6 \pm 1.4$ & $39.1 \pm 1.6$ & $8.3 \pm 0.1$ & 11.3 \\
\hline & Aerosol & $46.8 \pm 1.8$ & $33.6 \pm 1.4$ & $19.6 \pm 0.5$ & \\
\hline \multirow[t]{2}{*}{ Rod } & Drop-cast & $27.4 \pm 1.2$ & $23.4 \pm 0.8$ & $49.3 \pm 1.2$ & 37.4 \\
\hline & Aerosol & $34.0 \pm 2.1$ & $54.1 \pm 1.7$ & $11.8 \pm 0.1$ & \\
\hline
\end{tabular}

a Standard error from the mean value.

*Translocation from leaf to root. The \% difference is calculated by subtracting \% amount of gold present in the root of drop cast delivered plant from the aerosol sprayed plant root. 

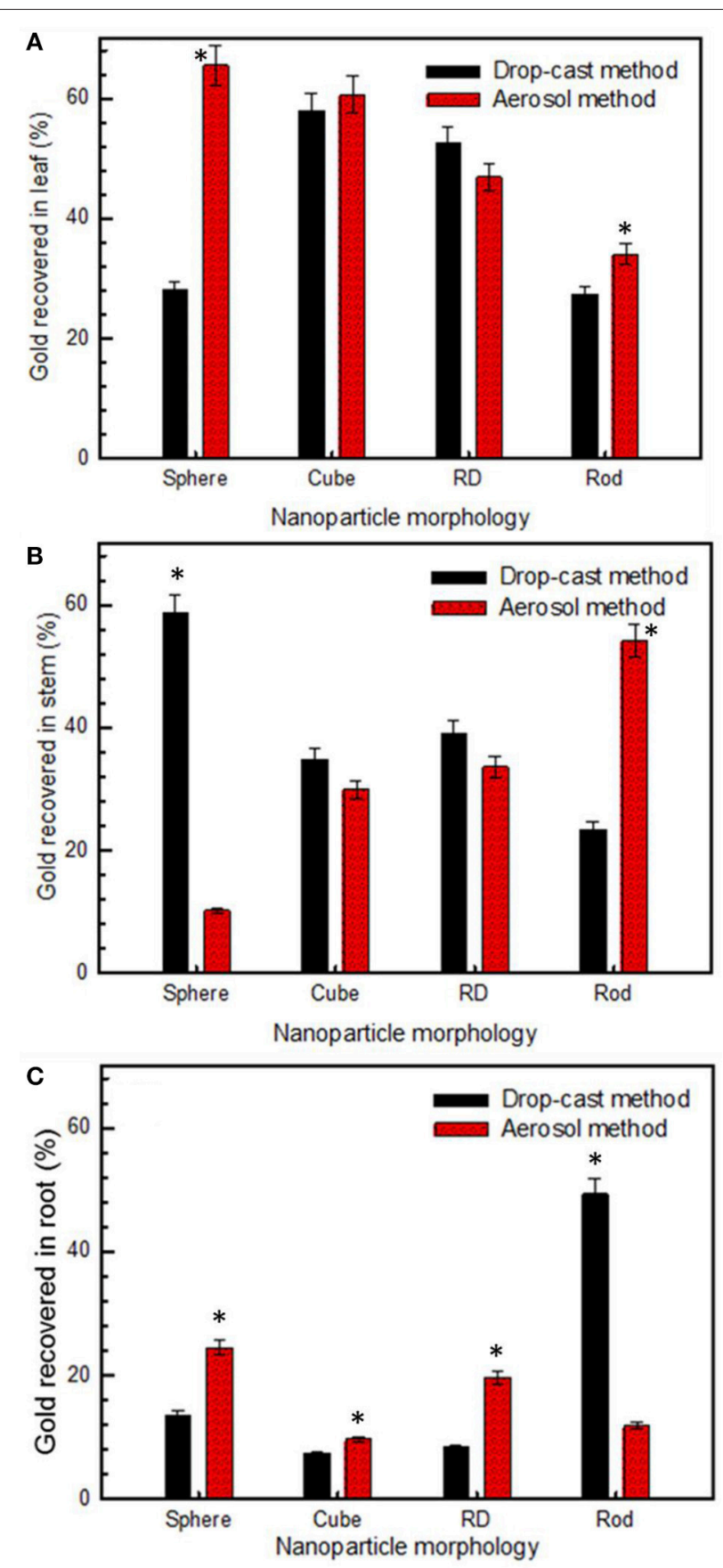

FIGURE 4 | Gold nanoparticles accumulation in watermelon that was recovered by ICP-MS in (A) leaf, (B) stem, and (C) root sections of treated plants, comparing results of drop-cast and aerosol method of nanoparticle delivery $(\boldsymbol{n}=\mathbf{4})$. Asterisk(s) above bar demonstrate significant difference $(p<0.05)$.

$(8.3 \%)>$ cube $(7.3 \%)$, whereas, trend for aerosol route applied particles were cube $(37.38 \%)>\mathrm{RD}(28.03 \%)>$ sphere $(17.76 \%)$ and rod $(16.82 \%)$. The results of the aerosol application method were further processed, normalizing the acquired concentration of gold by the online measurement of number concentration of particles in order to account for any discrepancies in the number of particles applied. From these normalized results (Figure 5A), a trend in the efficacy of translocation (number of particles recovered in root) was observed as: cube $(20,000)>\mathrm{RD}$ $(15,000)>$ sphere $(9500)>\operatorname{rod}(9000)$, a trend which again suggests that the aerosol application method results in improved translocation of low aspect ratio particles. In this experiment, we did not see any recognizable stress responses in the treated plants, supporting our hypothesis that AuNP are nontoxic to plants due to their biologically inert state (Shukla et al., 2005). Notwithstanding, our procedure required only $48 \mathrm{~h}$ of AuNP exposure; therefore, to further verify this hypothesis, extended toxicological studies must be conducted to determine the long-term effects of Au NPs on plants.

Foliar application increases uptake of nanoparticles by bypassing the cuticle, the primary barrier (Wang et al., 2013), although cuticle repels polar substances (Schwab et al., 2015). The stomatal pore in a typical plant leaf is approximately $100 \mathrm{~nm}$ in diameter (Schwab et al., 2015), presents a relatively large gap for the weak cuticle charge to sustain complete anion repulsion (Eichert and Goldbach, 2008). Aaerosol application method surpasses the cuticle barrier by delivering nanoparticles through the stomatal openings (Figure 5B). The subsequent transport of $\mathrm{Au}$ NPs from shoot to root is then achieved by plant's vascular systems, portrayed schematically in Figure 6. Cellular transport of nanoparticles carried out by both apoplast (through the wall) and symplast (cell to the cell, mediated by plasmodesmata). Apoplast pathway favors transport of larger particles $(\sim 200 \mathrm{~nm})$ but symplast pathway favors smaller $(<50 \mathrm{~nm})$ particles (Schwab et al., 2015). The cellular transport limitation suggests that apoplast pathway is dominant for drop cast methods, whereas, symplast is more common for aerosol mediated delivery of nanoparticles. It also explains the reason for more transport of rod shape particles to roots though drop cast than aerosol approach. The rod-shaped NPs that penetrated or were internalized in the cell have more probability to remain in the plasmodesmata due to the high aspect (inset of Figure 6). Once nanoparticles were internalized further, transport took place through the vascular system of the phloem. The pressure gradient of photosynthate in leaves driven a flow stream of nanoparticles and assist to move in phloem through phloem loading mechanism (Giaquinta, 1983). This pathway of nanoparticle transport through the xylem and phloem has been verified previously (Wang et al., 2012; Raliya et al., 2015). As a result of vascular transport of $\mathrm{Au}$ nanoparticles in watermelon plants, they were found to accumulate in the root, stem and leaf to varying degrees, both due to particle morphology as well as the nanoparticle delivery technique. The study opens the door for investigation to use compatible metal nanostructure for targeted delivery of genetic material to plants.

In summary, cellular uptake and accumulation of gold nanoparticles within C. lanatus was confirmed by ICP-MS after delivery through their leaf surface. Translocation of nanoparticles from leaf to root showed evidence that nanoparticles travel by the phloem transport mechanism. From the morphologydependent trends in nanoparticle translocation, it can be concluded that accumulation and transport of nanoparticles 

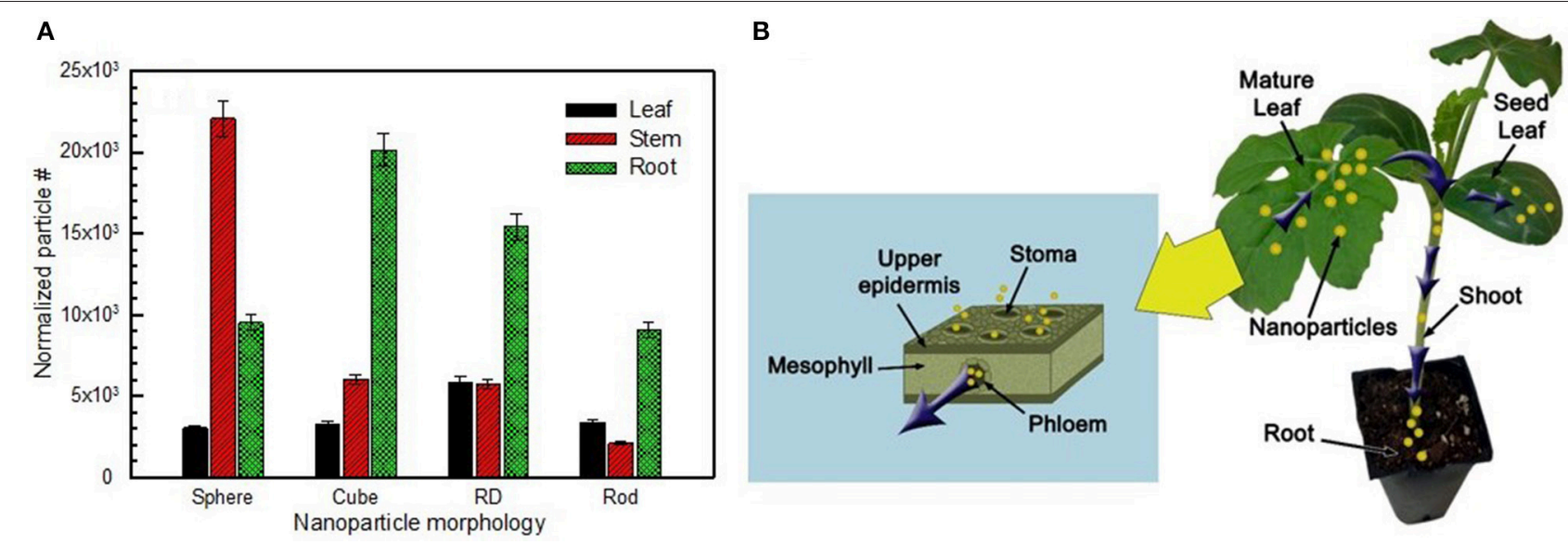

FIGURE 5 | Transport and accumulation of gold nanoparticles. (A) Number of particles recovered in sections of aerosol-treated plants, normalized to SMPS number concentration of particles. (B) Schematic of translocation of nanoparticles from leaf to root by phloem transport. Inset: gas phase uptake of nanoparticles through stomata opening.

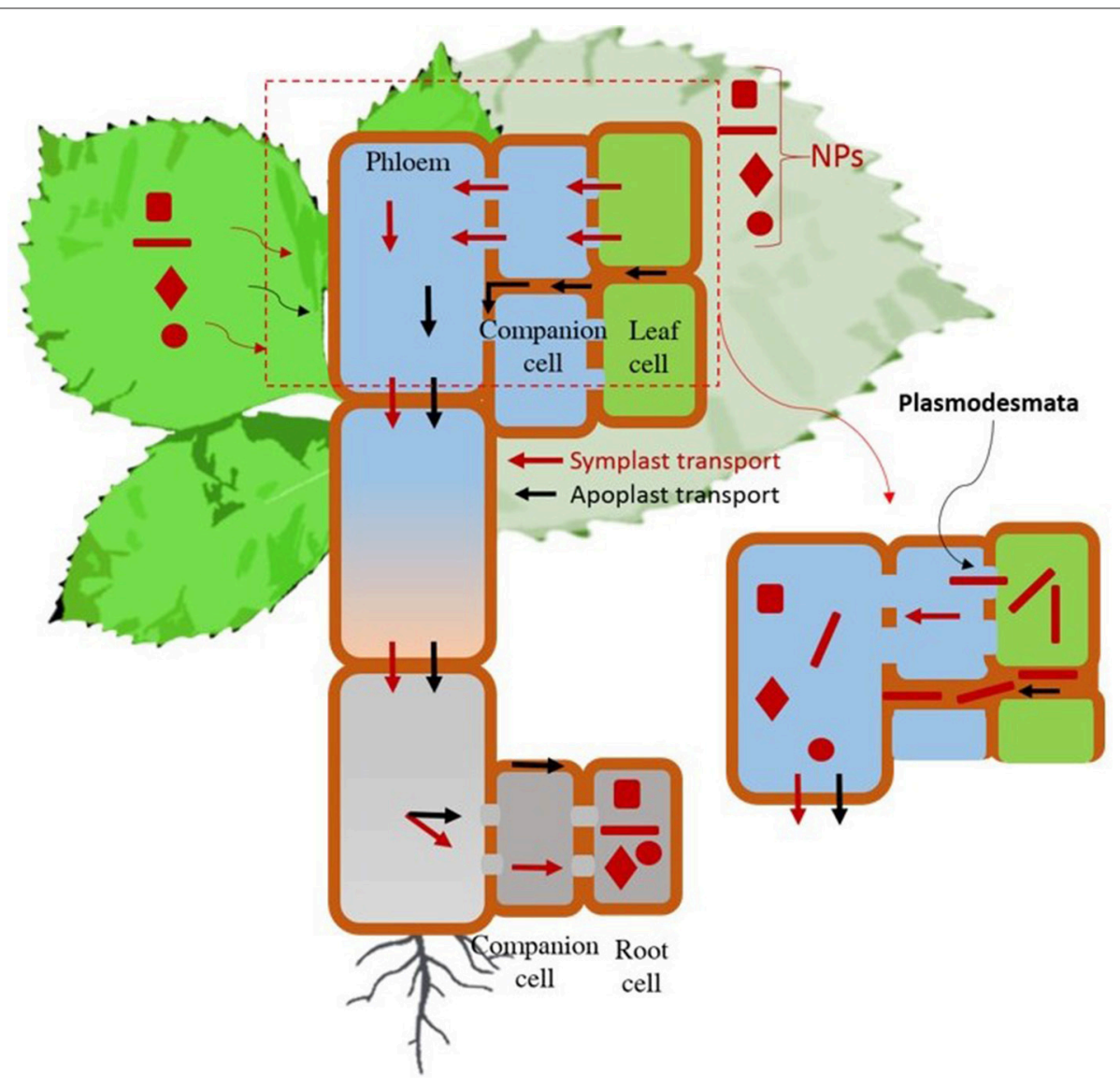

FIGURE 6 | Mechanistic understanding of nanoparticle transport within plant cells. Representation describes how nanoparticles transport through Apoplast and Symplast pathway in plants cells along with pressure gradient or mass flow of photosynthate product. Inset represent the favorable transport of gold nanostructure (rod shape) more through Apoplast than Symplastic pathway. NPs, nanoparticles. Color gradient in the phloem represents mass concentration of photosynthate with nanoparticles. 
depends on nanoparticle shape. Such trends were also influenced by the application method used. Application of nanoparticles by the aerosol method was most effective with structures with low aspect ratio, resulting in greater translocation than application by the drop-cast method. Conversely, the drop-cast method resulted in higher translocation of nanorods, which had a higher aspect ratio than other morphologies tested. This evidence suggests that different application methods may be optimal for delivery of different morphologies of nanoparticles to plants.

\section{AUTHOR CONTRIBUTIONS}

RR, CF did the plant exposure experiment. RR, CF and SC synthesize and characterize the gold nanoparticles. SC, CF, RN and NR contributed in ICP MS analyses. PB help in overall

\section{REFERENCES}

Albanese, A., Tang, P. S., and Chan, W. C. (2012). The effect of nanoparticle size, shape, and surface chemistry on biological systems. Ann. Rev. Biomed. Eng. 14, 1-16. doi: 10.1146/annurev-bioeng-071811-150124

Becker, R., Liedberg, B., and Kall, P.-O. (2010). CTAB promoted synthesis of Au nanorods - Temperature effects and stability considerations. J. Coll. Interf. Sci. 343, 25-30. doi: 10.1016/j.jcis.2009.10.075

Biswas, P., and Wu, C.-Y. (2005). Nanoparticles and the environment. J. Air Waste Manag. Assoc. 55, 708-746. doi: 10.1080/10473289.2005.10464656

Brandt, O., Rajathurai, A. M., and Roth, P. (1987). First observations on breakup of particle agglomerates in shock waves. Exp. Fluids 5, 86-94. doi: 10.1007/BF00776178

Capaldi Arruda, S. C., Diniz Silva, A. L., Moretto Galazzi, R., Antunes Azevedo, R., and Zezzi Arruda, M. A. (2015). Nanoparticles applied to plant science: a review. Talanta 131, 693-705. doi: 10.1016/j.talanta.2014.08.050

Chen, H., Seiber, J. N., and Hotze, M. (2014). ACS select on nanotechnology in food and agriculture: a perspective on implications and applications. J. Agri. Food Chem. 62, 1209-1212. doi: 10.1021/jf5002588

DeRosa, M. C., Monreal, C., Schnitzer, M., Walsh, R., and Sultan, Y. (2010). Nanotechnology in fertilizers. Nat. Nanotech. 5, 91-91. doi: 10.1038/nnano.2010.2

Eichert, T., and Goldbach, H. E. (2008). Equivalent pore radii of hydrophilic foliar uptake routes in stomatous and astomatous leaf surfaces - further evidence for a stomatal pathway. Physiol. Planta. 132, 491-502. doi: 10.1111/j.13993054.2007.01023.x

Ghosh, P. S., Kim, C.-K., Han, G., Forbes, N. S., and Rotello, V. M. (2008). Efficient gene delivery vectors by tuning the surface charge density of amino acid-functionalized gold nanoparticles. ACS Nano 2, 2213-2218. doi: $10.1021 / \mathrm{nn} 800507 \mathrm{t}$

Giaquinta, R. T. (1983). Phloem loading of sucrose. Ann. Rev. Plant Physiol. 34, 347-387. doi: 10.1146/annurev.pp.34.060183.002023

González-Melendi, P., Fernández-Pacheco, R., Coronado, M. J., Corredor, E., Testillano, P., Risueño, M. C., et al. (2008). Nanoparticles as smart treatment-delivery systems in plants: assessment of different techniques of microscopy for their visualization in plant tissues. Ann. Bot. 101, 187-195. doi: $10.1093 / \mathrm{aob} / \mathrm{mcm} 283$

Hautier, Y., Seabloom, E. W., Borer, E. T., Adler, P. B., Harpole, W. S., Hillebrand, H., et al. (2014). Eutrophication weakens stabilizing effects of diversity in natural grasslands. Nature 508, 521-525. doi: 10.1038/nature13014

Jiang, J., Oberdörster, G., Elder, A., Gelein, R., Mercer, P., and Biswas, P. (2008). Does nanoparticle activity depend upon size and crystal phase? Nanotoxicology 2, 33-42. doi: 10.1080/17435390701882478

Liu, R., and Lal, R. (2014). Synthetic apatite nanoparticles as a phosphorus fertilizer for soybean (Glycine max). Sci. Rep. 4:5686. doi: 10.1038/srep05686. Available online at: http://www.nature.com/articles/srep05686 experimental plan and suggestion that driven the study to conclusion. RR, CF wrote the manuscript and others reviewed the draft.

\section{ACKNOWLEDGMENTS}

This work was performed in part at the Nano Research Facility (NRF) of Washington University in St. Louis. This research facility was a member of the National Nanotechnology Infrastructure Network (NNIN) supported by the National Science Foundation under Grant No. CS-0335765. Partial support by the Lopata Endowment is gratefully acknowledged. CF appreciates the support from the National Nanotechnology Infrastructure Network Research Experience for Undergraduates (NNIN REU) -2015 program.

Liu, R., and Lal, R. (2015). Potentials of engineered nanoparticles as fertilizers for increasing agronomic productions. Sci. Total Env. 514, 131-139. doi: 10.1016/j.scitotenv.2015.01.104

Nair, R., Varghese, S. H., Nair, B. G., Maekawa, T., Yoshida, Y., and Kumar, D. S. (2010). Nanoparticulate material delivery to plants. Plant Sci. 179, 154-163. doi: 10.1016/j.plantsci.2010.04.012

Peng, J., Sun, Y., Liu, Q., Yang, Y., Zhou, J., Feng, W., et al. (2012). Upconversion nanoparticles dramatically promote plant growth without toxicity. Nano Res. 5, 770-782. doi: 10.1007/s12274-012-0261-y

Raliya, R., Nair, R., Chavalmane, S., Wang, W.-N., and Biswas, P. (2015). Mechanistic evaluation of translocation and physiological impact of titanium dioxide and zinc oxide nanoparticles on the tomato (Solanum lycopersicum L.) plant. Metallomics 7, 1584-1594. doi: 10.1039/C5MT00168D

Raliya, R., and Tarafdar, J. (2013). ZnO nanoparticle biosynthesis and its effect on phosphorous-mobilizing enzyme secretion and gum contents in Clusterbean (Cyamopsis tetragonoloba L.). Agr. Res. 2, 48-57. doi: 10.1007/s40003-01 2-0049-z

Raliya, R., Tarafdar, J. C., and Biswas, P. (2016). Enhancing the mobilization of native phosphorus in the Mung Bean Rhizosphere using ZnO Nanoparticles synthesized by soil Fungi. J. Agri. Food Chem. 64, 3111-3118. doi: 10.1021/acs.jafc.5b05224

Raliya, R., Tarafdar, J., Singh, S., Gautam, R., Choudhary, K., Maurino, V. G., et al. (2014). MgO Nanoparticles Biosynthesis and its effect on Chlorophyll contents in the leaves of Clusterbean (Cyamopsis tetragonoloba L.). Adv. Sci. Eng. Med. 6, 538-545. doi: 10.1166/asem.2014.1540

Saharan, V., Sharma, G., Yadav, M., Choudhary, M. K., Sharma, S., Pal, A., et al. (2015). Synthesis and in vitro antifungal efficacy of $\mathrm{Cu}$-chitosan nanoparticles against pathogenic fungi of tomato. Int. J. Biol. Macromol. 75, 346-353. doi: 10.1016/j.ijbiomac.2015.01.027

Scheffer, A., Engelhard, C., Sperling, M., and Buscher, W. (2008). ICP-MS as a new tool for the determination of gold nanoparticles in bioanalytical applications. Anal. Bioanalytic. Chem. 390, 249-252. doi: 10.1007/s00216-007-1576-5

Schwab, F., Zhai, G., Kern, M., Turner, A., Schnoor, J. L., and Wiesner, M. R. (2015). Barriers, pathways and processes for uptake, translocation and accumulation of nanomaterials in plants - Critical review. Nanotoxicology 10, 257-278. doi: 10.3109/17435390.17432015.11048326

Shukla, R., Bansal, V., Chaudhary, M., Basu, A., Bhonde, R. R., and Sastry, M. (2005). Biocompatibility of Gold Nanoparticles and their Endocytotic fate inside the cellular compartment: a microscopic overview. Langmuir 21, 10644-10654. doi: 10.1021/la0513712

Smith, V. H., Tilman, G. D., and Nekola, J. C. (1999). Eutrophication: impacts of excess nutrient inputs on freshwater, marine, and terrestrial ecosystems. Environ. Pollut. 100, 179-196. doi: 10.1016/S0269-7491(99)00091-3

Tarafdar, J., Raliya, R., Mahawar, H., and Rathore, I. (2014). Development of zinc nanofertilizer to enhance crop production in pearl millet (Pennisetum americanum). Agri. Res. 3, 257-262. doi: 10.1007/s40003-014-0113-y 
Thakor, A. S., Jokerst, J., Zavaleta, C., Massoud, T. F., and Gambhir, S. S. (2011). Gold nanoparticles: a revival in precious metal administration to patients. Nano Lett. 11, 4029-4036. doi: 10.1021/nl202559p

Wang, W.-N., Tarafdar, J. C., and Biswas, P. (2013). Nanoparticle synthesis and delivery by an aerosol route for watermelon plant foliar uptake. J. Nanopart. Res. 15, 1413-1417. doi: 10.1007/s11051-013-1417-8

Wang, Z., Xie, X., Zhao, J., Liu, X., Feng, W., White, J. C., et al. (2012). Xylem- and phloem-based transport of $\mathrm{CuO}$ nanoparticles in maize (Zea mays L.). Env. Sci. Technol. 46, 4434-4441. doi: 10.1021/es204212z

Wei, J., Liu, H., Liu, F., Zhu, M., Zhou, X., and Xing, D. (2014). Miniaturized paper-based gene sensor for rapid and sensitive identification of contagious plant virus. ACS Appl. Mat. Interf. 6, 22577-22584. doi: 10.1021/am506695g

Wibowo, D., Zhao, C. X., Peters, B. C., and Middelberg, A. P. J. (2014). Sustained release of fipronil insecticide in vitro and in vivo from biocompatible Silica Nanocapsules. J. Agri. Food Chem. 62, 12504-12511. doi: 10.1021/jf504455x

Wu, H.-L., Kuo, C.-H., and Huang, M. H. (2010). Seed-mediated synthesis of Gold Nanocrystals with systematic shape evolution from Cubic to Trisoctahedral and Rhombic Dodecahedral structures. Langmuir 26, 12307-12313. doi: 10.1021/la1015065
Yang, Y., Wang, Y., Westerhoff, P., Hristovski, K., Jin, V. L., Johnson, M.-V. V., et al. (2014). Metal and nanoparticle occurrence in biosolid-amended soils. Sci. Total Environ. 485, 441-449. doi: 10.1016/j.scitotenv.2014.03.122

Zhao, W., Lu, J., Ma, W., Xu, C., Kuang, H., and Zhu, S. (2011). Rapid onsite detection of Acidovorax avenae subsp. citrulli by gold-labeled DNA strip sensor. Biosens. Bioelectron. 26, 4241-4244. doi: 10.1016/j.bios.2011. 04.004

Conflict of Interest Statement: The authors declare that the research was conducted in the absence of any commercial or financial relationships that could be construed as a potential conflict of interest.

Copyright (C) 2016 Raliya, Franke, Chavalmane, Nair, Reed and Biswas. This is an open-access article distributed under the terms of the Creative Commons Attribution License (CC BY). The use, distribution or reproduction in other forums is permitted, provided the original author(s) or licensor are credited and that the original publication in this journal is cited, in accordance with accepted academic practice. No use, distribution or reproduction is permitted which does not comply with these terms. 